\title{
Analysis of Impact of Tuned Damper Floating Fastener on Track System
}

\author{
Anbin Wang, Zheyu Zhang and Zhiqiang Wang \\ Railway Research Center, Luoyang Ship Material Research Institute, Luoyang 471023, China
}

\begin{abstract}
Ground-borne noise caused by track vibration became one of major problem affecting urban rail transit development. Resilient rail fasteners come to be a main measure of reducing ground-borne noise. Low stiffness of fastener contributes to ensuring the good effect of vibration isolation, but causing higher vibration of rails, while the tuned damper fastener can make it up. In this paper, the simulation of rail receptance is used to fit on site measured data, then the data are used to build a vehicle-track-foundation model to do the calculations, like vibration reduction of track systems and rails. According to the results, the tuned damper fastener can reduce the vibration of slab as well as rails.
\end{abstract}

Key words: Vibration reduction, tuned damper fastener, simulation.

\section{Introduction}

The vibration of track system becomes one of the major problems affecting urban rail transit development, with the rapid development of rail transit; measures of rail vibration and noise reduction increasingly attract focus [1]. Setting rail fasteners for vibration reduction is one of the main damping measures in current rail track systems. Rail fasteners are located between the rail and sleepers, connecting and fixing the rail at the same time reducing the vibration transmission to the ballast and foundation. Lower the vertical stiffness is, better the effect of vibration isolation is. Current track traffic conditions are increasingly diverse, while the environmental requirements are higher and higher. Ordinary rail fasteners (vertical stiffness range: 40-200 MN/m), even medium rail fasteners (vertical stiffness range: $15-40 \mathrm{MN} / \mathrm{m}$ ) all have been unable to meet the high vibration reduction requirements (10-15 dB), so rail fastener with low vertical stiffness in recent years to attract more and more attention.

Although the better vibration isolation performance

Corresponding author: Zheyu Zhang, engineer, master, research field: noise and vibration reduction in railway. can be achieved, a new problem has been produced by low stiffness, which may be in some cases, the rail vibration will be increased in a certain extent, thus easily lead to rail corrugation, noise increasing. The setting of the anti-resonant unit can effectively solve this problem; part of the rail vibration can be dissipated through the anti-resonant unit, so that the rail vibration can be reduced.

In this paper, the finite element analysis has been adopted to analyze the track dynamics and vibration reduction effect of low stiffness and tuned damper fastener (referred to as the "tuned damper fastener") on rail and slab track system. It can be seen that the tuned damper fastener can significantly reduce the vibration of the track system and effectively suppress the vibration of the rail at the same time.

\section{The Structure and Characteristics of the Tuned Damper Fastener}

Tuned damper fastener holds the rail by two elastomeric support blocks against to the rail on both sides so that the rail can be "floating" in the vertical direction as shown in Fig. 1. The low stiffness can be achieved to get a better vibration isolation performance. Constrained position of rail moves from rail foot to rail 


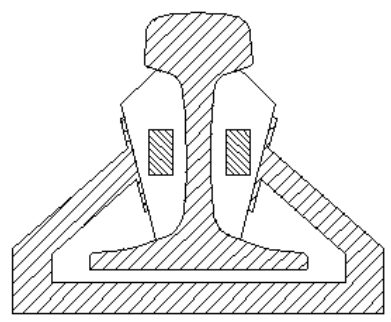

Fig. 1 Model of the tuned damper fastener.

web and rail head so that the lateral stiffness will not significantly reduce to meet the track safety requirement of vehicle operation.

An anti-resonant system is set in the support block which can be taken as a dynamic vibration absorber. It can effectively dissipate the vibration energy of the rail, thereby reducing the radiation of the rail noise, the rail corrugation and rail-wheel force [2].

\section{Analysis of Vibration Reduction Performance of Tuned Damper Fastener}

Tuned damper fastener has a low vertical stiffness, compared with conventional fastener with normal stiffness range of $40-200 \mathrm{MN} / \mathrm{m}$. It has significant vibration isolation effect in the frequency bands upon the "P2" frequency. In this paper, a finite element method is used though the establishment of the model with rail, fastener, slab and foundation to calculate the vibration reduction effect of the tuned damper fastener compared with the conventional fastener on rails, ballasts and foundations.

\subsection{Fitting of Fastener Parameters}

In order to eliminate the effect of the different factors, such as the axle load, vehicle speed, curve radius and the wheel rail surface conditions of measurement results, frequency response functions of the track system can be used. The frequency response function can identify system's natural characteristics such as track stiffness, resonance frequencies, etc.

3.1.1 On Site Measure Method of Frequency Response Functions

Measure method in Ref. [3] is used, and Fig. 2 shows the diagrammatic drawing of track frequency response
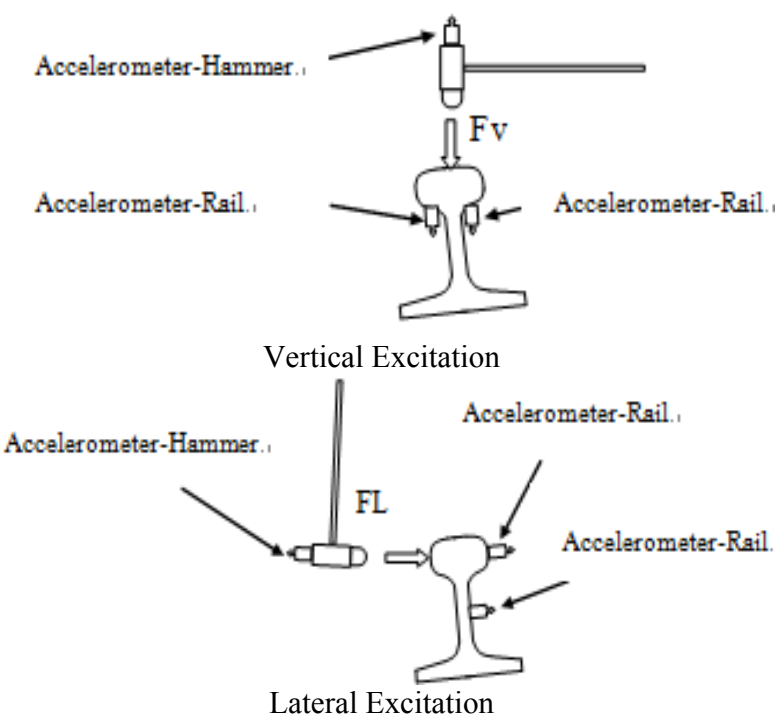

Fig. 2 Track frequency response function test [3].

function tests. The positions of excitation and response can either located at the middle span of the two fasteners and or directly over the fasteners. According to fitting the result, characteristics parameters of track system, such as support stiffness, damping loss factor, and so on, can be obtained.

3.1.2 Comparison between Simulation and Measurement

Rails are modelled by beam element with parameters of $60 \mathrm{~kg}$ rail. Figs. 3-6 show the rail receptance spectrum just above the support and at the middle span of adjacent fasteners of two kinds of fasteners in the vertical and lateral directions respectively. Theoretical calculations and field testing results are drawn on the same graph. It can be seen that measurement and prediction are in a good agreement. The theoretical predicted and tested parameters of two kinds of fasteners in the vertical direction are given in Tables 1 and 3 , and the equivalent lateral parameters are shown in Tables 2 and 4, which are corresponding to lateral receptance at the position at the rail head.

Vibration value in the vertical direction is always taken as the indicator for measuring the vibration level of the track system [4], so in the following parts, the vibration reduction performance of different fasteners are valued by the vertical vibration amount of rail, slab and foundation. 


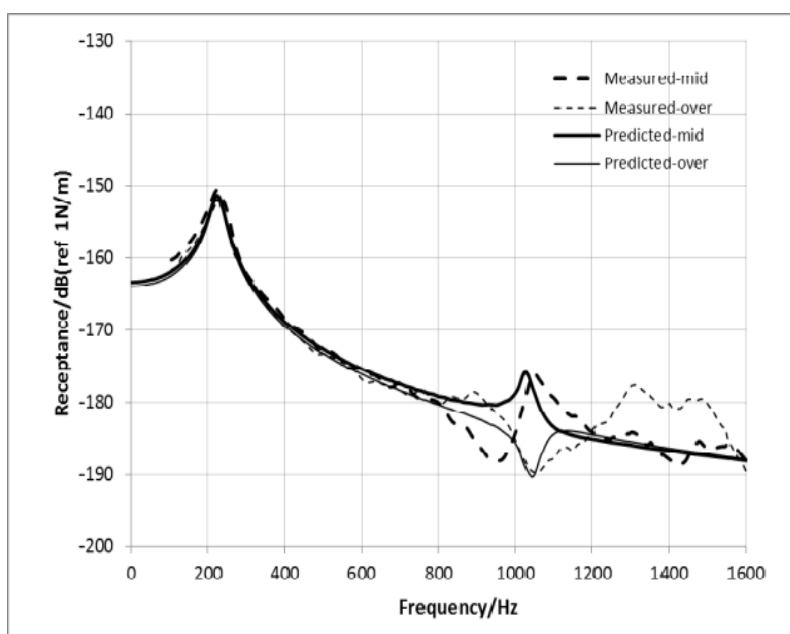

Fig. 3 Rail receptance in vertical direction of the common fastener.

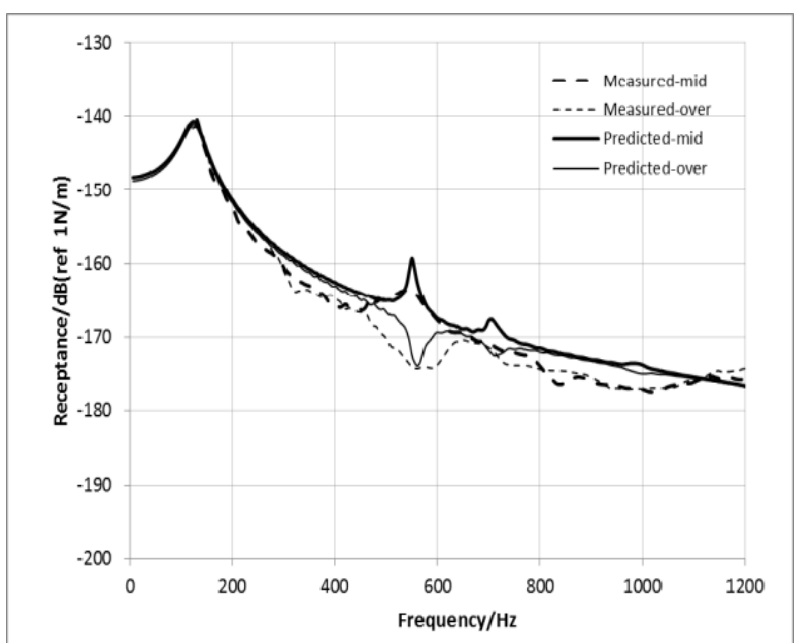

Fig. 4 Rail receptance in lateral direction of the common fastener.

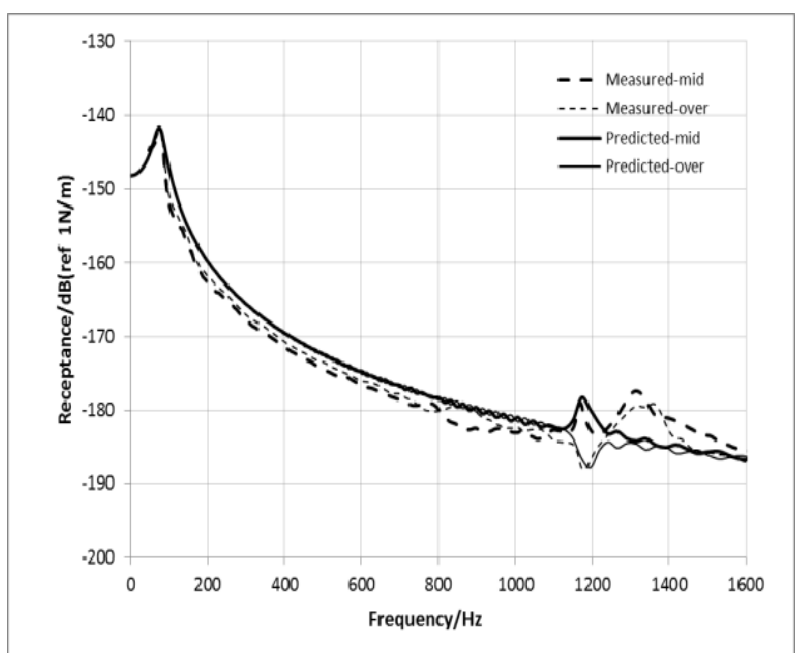

Fig. 5 Rail receptance in vertical direction of the tuned damper fastener.

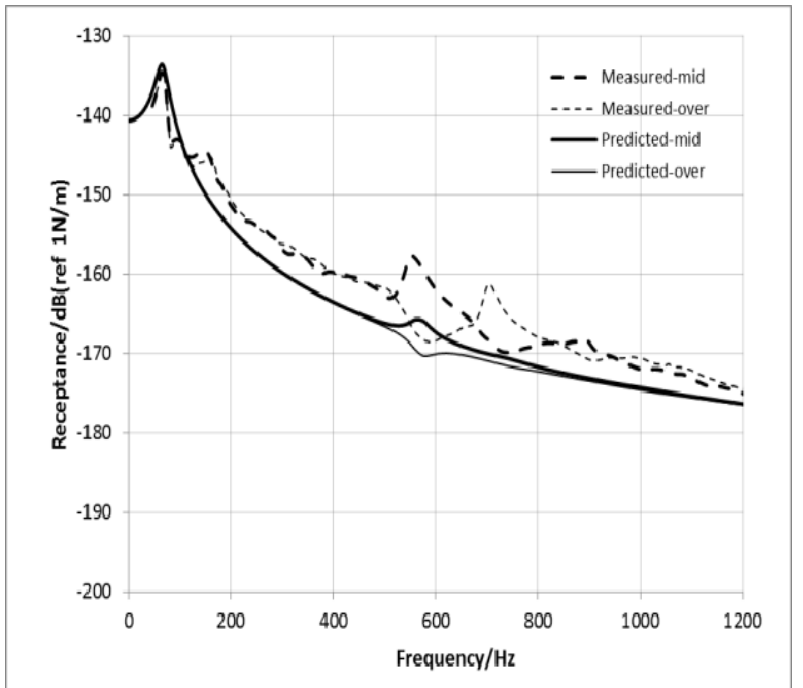

Fig. 6 Rail receptance in lateral direction of the tuned damper fastener.

Table 1 Track parameters in vertical direction.

\begin{tabular}{ll}
\hline Item & Value \\
\hline (A): Equivalent density: $\rho \mathrm{A} /(\mathrm{kg} / \mathrm{m})$ & 60.4 \\
(B): Vertical support stiffness: $\mathrm{k} /(\mathrm{MN} / \mathrm{m})$ & 75 \\
(C): Support spacing: $1 / \mathrm{m}$ & 0.625 \\
(D): Poisson ratio: $v$ & 0.3 \\
\hline
\end{tabular}

Table 2 Track parameters in lateral direction.

\begin{tabular}{ll}
\hline Item & Value \\
\hline (A): Equivalent density: $\rho \mathrm{A} /(\mathrm{kg} / \mathrm{m})$ & 39.3 \\
(B): Vertical support stiffness: $\mathrm{k} /(\mathrm{MN} / \mathrm{m})$ & 13 \\
(C): Support spacing: $1 / \mathrm{m}$ & 0.625 \\
(D): Poisson ratio: $v$ & 0.3 \\
\hline
\end{tabular}

Table 3 Track parameters in vertical direction.

\begin{tabular}{ll}
\hline Item & Value \\
\hline (A): Equivalent density: $\rho \mathrm{A} /(\mathrm{kg} / \mathrm{m})$ & 60.4 \\
(B): Vertical support stiffness: $\mathrm{k} /(\mathrm{MN} / \mathrm{m})$ & 8.5 \\
(C): Support spacing: $1 / \mathrm{m}$ & 0.625 \\
(D): Poisson ratio: $v$ & 0.3 \\
\hline
\end{tabular}

Table 4 Track parameters in lateral direction.

\begin{tabular}{ll}
\hline Item & Value \\
\hline (A): Equivalent density: $\rho \mathrm{A} /(\mathrm{kg} / \mathrm{m})$ & 35 \\
(B): Vertical support stiffness: $\mathrm{k} /(\mathrm{MN} / \mathrm{m})$ & 3.75 \\
(C): Support spacing: $1 / \mathrm{m}$ & 0.625 \\
(D): Poisson ratio: $v$ & 0.3 \\
\hline
\end{tabular}

3.2 Analysis of Vibration Reduction Effect of Tuned Damper Fastener on Track System

\subsubsection{Track System Simulation Model}

In recent years, slab track is widely used in subways, which is formed by rails, slabs, CA mortar layer and 
concrete base. In the simulation model, rails are built by beam elements, track system is built by hexahedral elements, and fasteners are simplified by springs. Material parameters are shown in Table 5, and slab dimensions are from Ref. [5], shown in Table 6.

\subsubsection{Artificial Boundaries of Foundation}

To calculate the ground vibration caused by track system, the foundation should be considered as infinite but limited by simulation efficiency, so that only finite foundation can be built, and the boundaries need to be set properly [6].

Consistent viscous-spring artificial boundaries are chosen, which set equivalent continuous distributed spring-damp system to simulate the infinite boundary. Normal and tangential spring stiffness and damping coefficient are in accordance with the following Eqs. (1) and (2) [7].

$$
\begin{aligned}
& K_{\mathrm{BN}}=\alpha_{\mathrm{N}} \frac{G}{R}, C_{\mathrm{BN}}=\rho C_{\mathrm{p}} \\
& K_{\mathrm{BT}}=\alpha_{\mathrm{T}} \frac{G}{R}, C_{\mathrm{BT}}=\rho C_{\mathrm{s}}
\end{aligned}
$$

where, $K_{\mathrm{BN}}, K_{\mathrm{BT}}$ represent spring stiffness, the subscript $\mathrm{N}$ and $\mathrm{T}$, respectively represent the normal and tangential direction; $C_{\mathrm{BN}}, C_{\mathrm{BT}}$ represent damping coefficient, the subscript $\mathrm{N}$ and $\mathrm{T}$ respectively represent the normal and tangential direction; $R$ represents the distance from the artificial boundary to source; $C_{\mathrm{p}}, C_{\mathrm{s}}$ represent medium wave velocity; $\rho$ represents medium density; $\alpha_{\mathrm{N}}, \alpha_{\mathrm{T}}$ represent factors, taking recommended value [7], as 1.33 and 0.67 .

\subsubsection{Comparison of Vibration Reduction Effect}

Simulation model is shown in Fig. 7, to consider the

Table 5 Material parameters.

\begin{tabular}{llll}
\hline Item & $\begin{array}{l}\text { Density/ } \\
\left(\mathrm{kg} \cdot \mathrm{m}^{-3}\right)\end{array}$ & $\begin{array}{l}\text { Modulus of } \\
\text { elasticity/MPa }\end{array}$ & Poisson ratio \\
\hline (A):Rail & 7850 & 210000 & 0.3 \\
(B):Sleeper & 2500 & 35000 & 0.15 \\
(C):Slab & 2500 & 32500 & 0.2 \\
(D):Ballast & 2500 & 20000 & 0.2 \\
(E):Mortar & 1500 & 300 & 0.34 \\
(F):Foundation & 1500 & 2000 & 0.25 \\
\hline
\end{tabular}

Table 6 Material parameters.

\begin{tabular}{llll}
\hline Item & Width $/ \mathrm{mm}$ & Length $/ \mathrm{mm}$ & Height $/ \mathrm{mm}$ \\
\hline (A):Slab & 2600 & 25000 & 240 \\
(B):Concrete & 800 & 300 & 150 \\
block & & 25000 & 300 \\
(C):Ballast & 3400 & & \\
\hline
\end{tabular}

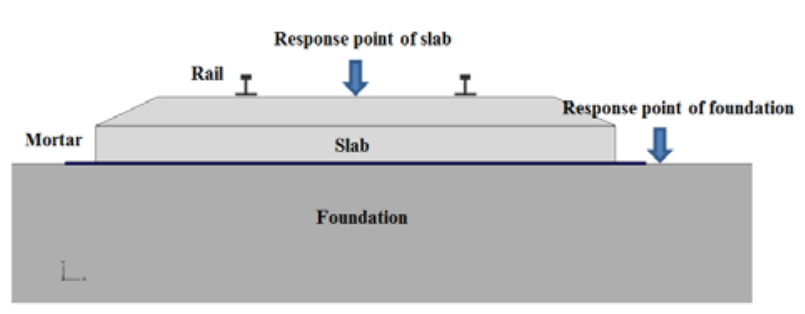

Fig. 7 Simulation model of track system.

effect of vehicle on track system, the unsprung mass of $850 \mathrm{~kg}$ is adopted. The response position of the slab is on the centre of the slab, and the one on foundation is $300 \mathrm{~mm}$ to the edge of slab, same as on-line measurement.

Figs. 8 and 9 are calculation results of vertical 1/3 octave spectrum and total vibration of slab. From the figures, the "P2" resonant frequency of tuned damper fastener (around $25 \mathrm{~Hz}$ ) is much lower than the one of conventional fastener (around $60 \mathrm{~Hz}$ ), significantly expanding the vibration isolation frequency range. The vertical vibration insertion loss of $13.7 \mathrm{~dB}$ of the slab has been achieved by the tuned damper fastener in frequency range of 1-80 Hz.

Figs. 10 and 11 are calculation results of vertical 1/3 octave spectrum and total vibration of foundation. The

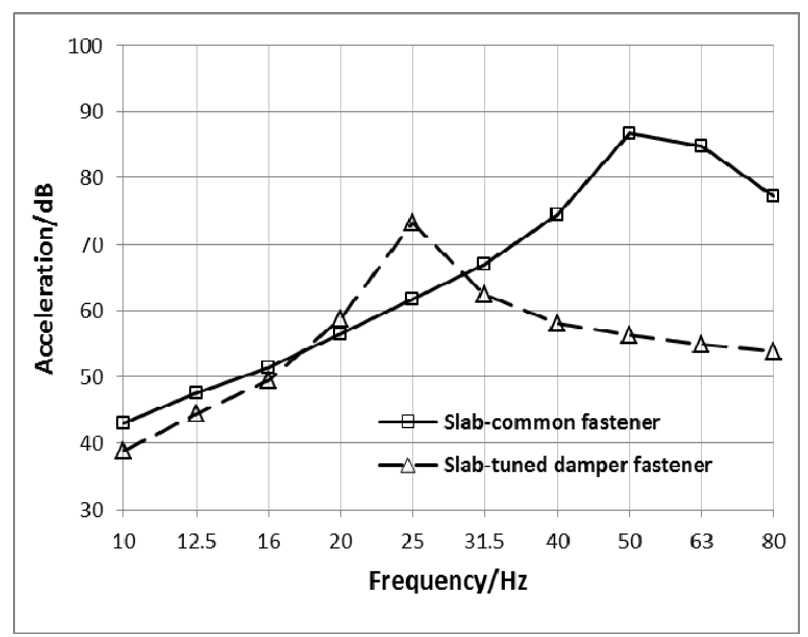

Fig. $8 \quad 1 / 3$ octave spectrum of slab. 


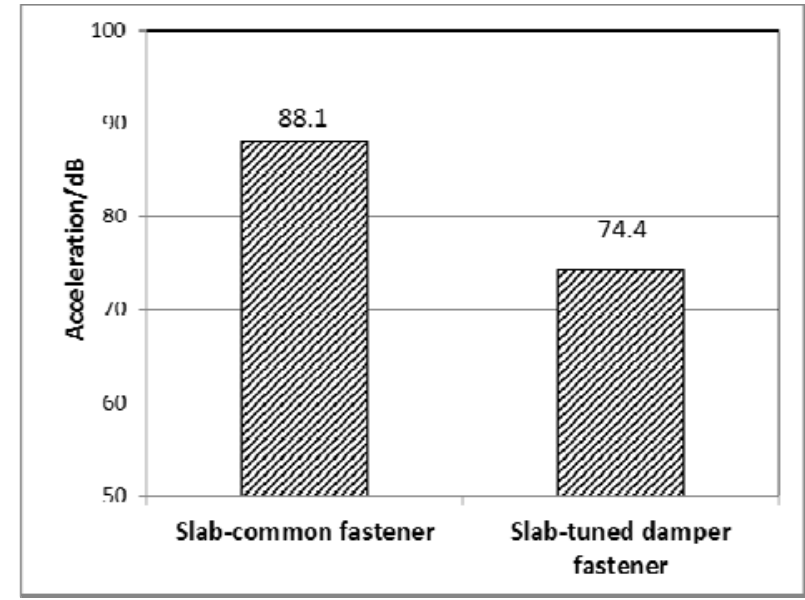

Fig. 9 Total vibration of slab $(1-80 \mathrm{~Hz})$.

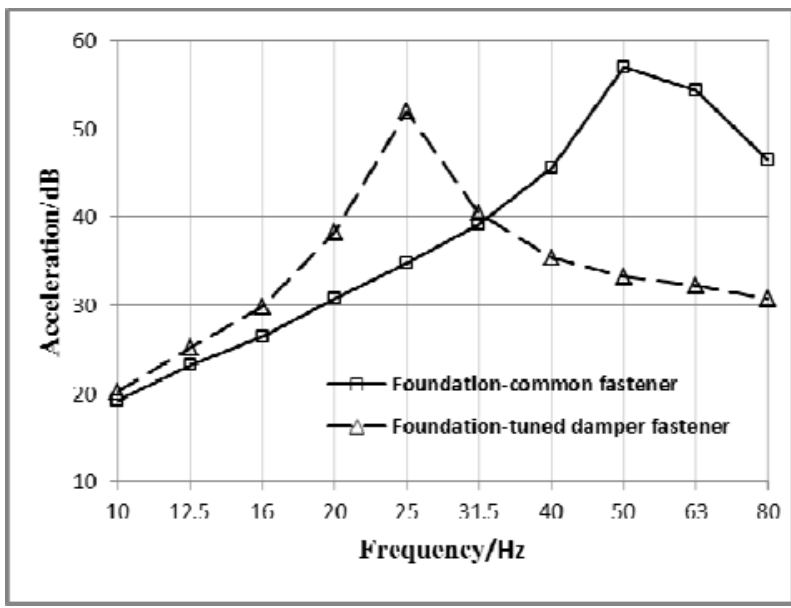

Fig. $101 / 3$ octave spectrum of foundation.

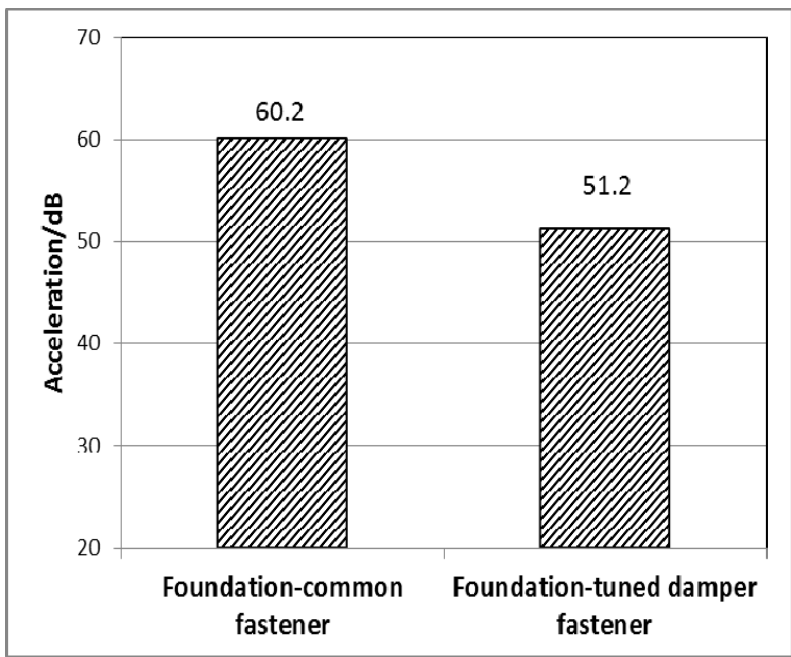

Fig. 11 Total vibration of foundation $(1-80 \mathrm{~Hz})$.

vertical vibration insertion loss of $9 \mathrm{~dB}$ of the foundation has been achieved by the tuned damper fastener in frequency range of 1-80 Hz.
3.3 Analysis of Vibration Reduction Effect of Tuned Damper Fastener on Rails

The lower the fastener stiffness is, the better vibration isolation effect is, but causing the rail vibration increasing [8], which leads to the increase of noise and rail corrugation. The "anti-resonant unit" is set to the tuned damper fastener, to dissipate vibration energy of rails in a certain extent.

Due to a variety of modes of the resonance unit in the vibration calculation frequency range $(12.5-2,000 \mathrm{~Hz})$, the beam-spring-mass models are too simple to simulate, so the solid model is used. Tuned damper fasteners are simplified as support blocks and the backboards. According to the fixing way, the backboards are fixed, and the support blocks and rail contact, as shown in Fig. 12. The rail vibration results of fasteners with or without anti-resonant unit are compared in Figs. 13 and 14.

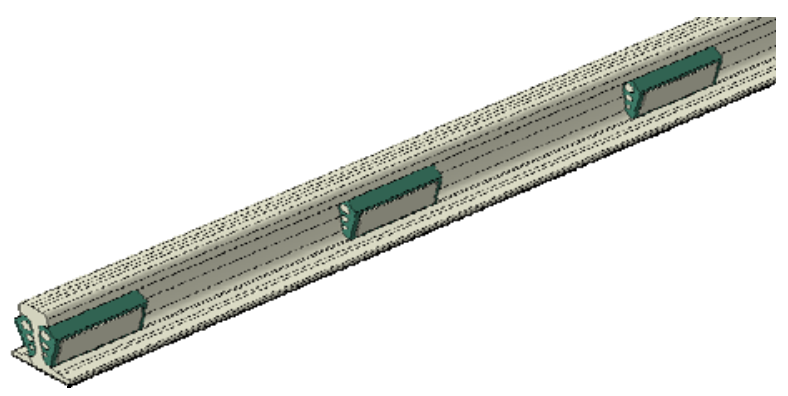

Fig. 12 Simulation model of rail vibration.

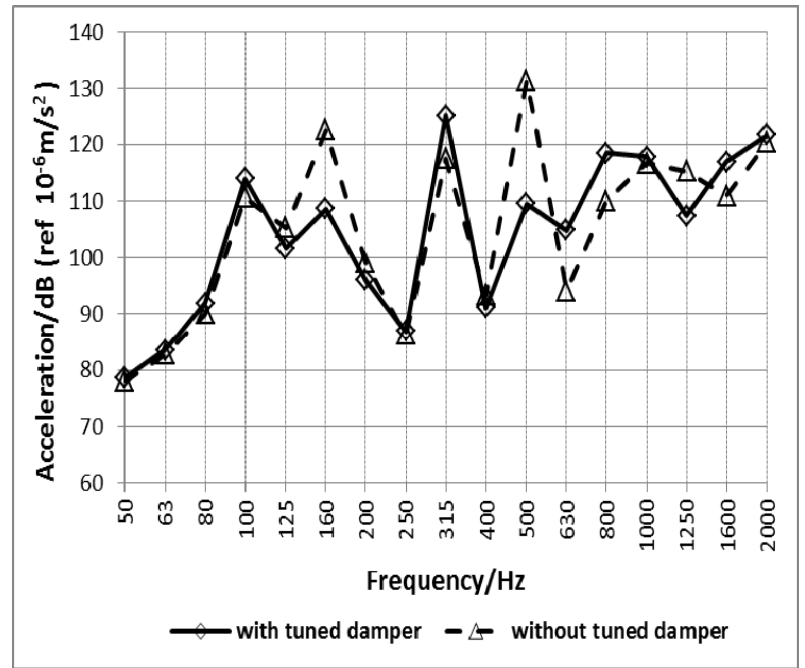

Fig. 13 1/3 octave spectrum of rail. 


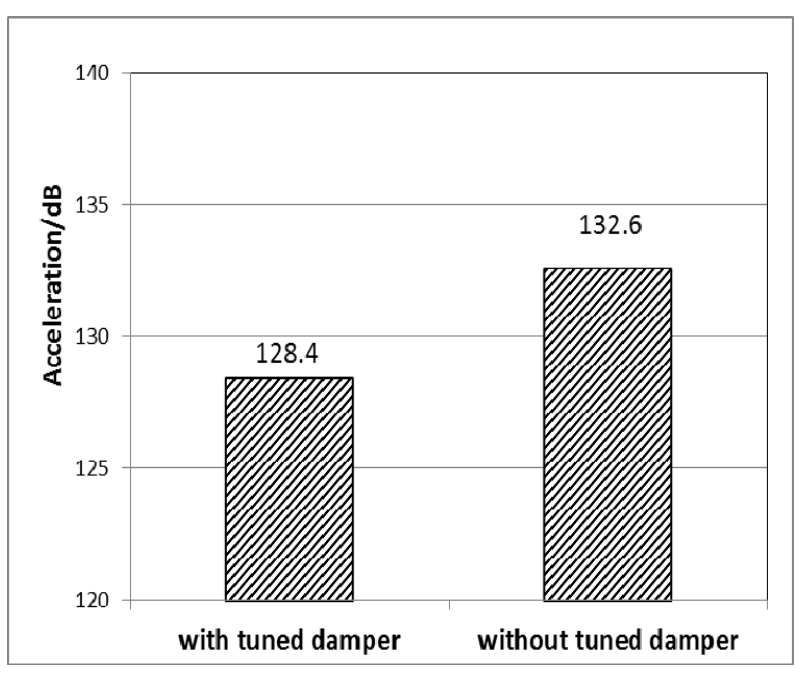

Fig. 14 Total vibration of rail $(12.5-2,000 \mathrm{~Hz})$.

From Figs. 13 and 14, it can be seen that the rail vibration decreases by $4.2 \mathrm{~dB}$ in the frequency range of $12.5-2,000 \mathrm{~Hz}$ with anti-resonant unit. Resonance effects are obvious at $160 \mathrm{~Hz}, 500 \mathrm{~Hz}$ and $1,250 \mathrm{~Hz}$, especially at $500 \mathrm{~Hz}$, with the vibration reduction of about $20 \mathrm{~dB}$. Fig. 5 shows that the "Pinned-Pinned" frequency of track system with tuned damper fasteners is around $1,200 \mathrm{~Hz}$, so the tuned damper fasteners can effectively reduce the rail corrugation [9].

\section{Conclusions}

In this paper, the effects of low stiffness and tuned damper fastener on track system are analyzed by means of numerical simulation, and the following conclusions are obtained:

(1) Low stiffness and tuned damper fastener (tuned damper fastener) has good isolation effect, and can effectively reduce the vibration transferring to the slab and foundation. Compared to the ordinary fasteners (dynamic stiffness about $75 \mathrm{MN} / \mathrm{m}$ ), in the range of 1-80 $\mathrm{Hz}$, the tuned damper fastener can reduce slab vibration of $13.7 \mathrm{~dB}$ and foundation vibration of $9 \mathrm{~dB}$.

(2) Low stiffness and tuned damper fastener (tuned damper fastener) can reduce rail vibration $4.2 \mathrm{~dB}$ in the range of $12.5-2,000 \mathrm{~Hz}$, especially obvious at $160 \mathrm{~Hz}$, $500 \mathrm{~Hz}$ and $1,250 \mathrm{~Hz}$, which contain the "Pinned-Pinned" frequency, thereby inhibiting steel rail corrugation.

\section{References}

[1] Gu, A. J., Liu, W. N., and Zhang, H. G. 2011. "Impact of Rail Fastenings' Stiffness and Damping on Abnormal Rail Corrugation." Urban Rapid Rail Transit 6: 1-4.

[2] Wang, Z. Q., Wang, A. B., and Bai, J. 2014. "Introduction and Experimental Analysis of Floating Rail Fasteners with Tuned Dampers." Noise and Vibration Control 34: 188-91.

[3] Wang, A. B., Wang, Z. Q., and Zhang, P. 2014. “The Mechanism of Rail Corrugation Generation and Development-Variation of Difference between Wheel and Rail Receptances." Development and Application of Materials 6: 6-15.

[4] NEPA. 1988. Standard of Vibration in Urban Area Environment [GB10071].

[5] Liang, Q. D. 2011. "Finite Element Analysis of Deformation Performance of Double Block Ballastless Track Slab." Master of Civil Engineering, Graduate Program in Centre South University.

[6] Wang, L. 2008. "Number Analysis of Ground Vibration Induced By Meglev Train Running in Tunnel.” Master of Vehicle Engineering, Graduate Program in Southwest Jiaotong University.

[7] Liu J. B., Wang Z. Y., Du X. L., and Du, X. Y. 2005. "Three-Dimensional Visco-Elastic Artificial Boundaries in Time Domain for Wave Motion Problems." Engineering Mechanics 22: 46-51.

[8] Yadav, S., and Doumanidis, C. 2005. "Thermomechanical Analysis of an URM (Ultrasonic Rapid Manufacturing) System.” Journal of Manufacturing Processes 7: 153-61. Wu, X.Y., Deng, J. 2013. "Rail Vibration Simulation Analysis with Different Elastic Fastener". Modern Urban Transit, (4): 44-51.

[9] Wang, A. B., Wang, Z. Q., Zhang, P., et al. 2015. "The Mechanism of Rail Corrugation and Development-Vibration of Difference between Wheel and Rail Reacceptance." Presented at the 22th International Congress on Sound and Vibration. 